\title{
Modeling Transposition of the Great Arteries with Patient-Specific Induced Pluripotent Stem Cells
}

\author{
Imelda Ontoria-Oviedo ${ }^{1, *(\mathbb{C}, \text { Gabor Földes }}{ }^{2,3}$, Sandra Tejedor ${ }^{1}{ }^{(}$, Joaquín Panadero ${ }^{4}$, Tomoya Kitani ${ }^{5}(\mathbb{D}$, \\ Alejandro Vázquez ${ }^{1}$, Joseph C. Wu ${ }^{5}$, Sian E. Harding ${ }^{2}\left(\mathbb{D}\right.$ and Pilar Sepúlveda ${ }^{1, *(1)}$ \\ 1 Regenerative Medicine and Heart Transplantation Unit, Instituto de Investigación Sanitaria La Fe, \\ 46026 Valencia, Spain; sandra.tejedorgascon@gmail.com (S.T.); avazsan@gmail.com (A.V.) \\ 2 National Heart and Lung Institute, Imperial College London, London W12 0NN, UK; \\ g.foldes@imperial.ac.uk (G.F.); sian.harding@imperial.ac.uk (S.E.H.) \\ 3 Heart and Vascular Center, Semmelweis University, H1122 Budapest, Hungary \\ 4 IGENOMIX S.L., Edificios Europark, Parque Tecnológico, 46980 Paterna, Spain; btcpar@gmail.com \\ 5 Stanford Cardiovascular Institute, Stanford University School of Medicine, Stanford, CA 94305, USA; \\ t-kitani@koto.kpu-m.ac.jp (T.K.); joewu@stanford.edu (J.C.W.) \\ * Correspondence: imeldaontoria@gmail.com (I.O.-O.); pilar.sepulveda.sanchis@gmail.com (P.S.); \\ Tel.: +34-96-1246632 (I.O.-O.); +34-96-1246635 (P.S.)
}

check for

updates

Citation: Ontoria-Oviedo, I.; Földes, G.; Tejedor, S.; Panadero, J.; Kitani, T.; Vázquez, A.; Wu, J.C.; Harding, S.E.; Sepúlveda, P. Modeling Transposition of the Great Arteries with Patient-Specific Induced Pluripotent Stem Cells. Int. J. Mol. Sci. 2021, 22, 13270. https://doi.org/10.3390/ ijms222413270

Academic Editor: Robert David

Received: 10 November 2021

Accepted: 7 December 2021

Published: 9 December 2021

Publisher's Note: MDPI stays neutral with regard to jurisdictional claims in published maps and institutional affiliations.

Copyright: (c) 2021 by the authors. Licensee MDPI, Basel, Switzerland. This article is an open access article distributed under the terms and conditions of the Creative Commons Attribution (CC BY) license (https:// creativecommons.org/licenses/by/ $4.0 /)$.

\begin{abstract}
The dextro-transposition of the great arteries (d-TGA) is one of the most common congenital heart diseases. To identify biological processes that could be related to the development of d-TGA, we established induced pluripotent stem cell (iPSC) lines from two patients with d-TGA and from two healthy subjects (as controls) and differentiated them into endothelial cells (iPSC-ECs). iPSC-EC transcriptome profiling and bioinformatics analysis revealed differences in the expression level of genes involved in circulatory system and animal organ development. iPSC-ECs from patients with d-TGA showed impaired ability to develop tubular structures in an in vitro capillary-like tube formation assay, and interactome studies revealed downregulation of biological processes related to Notch signaling, circulatory system development and angiogenesis, pointing to alterations in vascular structure development. Our study provides an iPSC-based cellular model to investigate the etiology of d-TGA.
\end{abstract}

Keywords: great arteries transposition; endothelial cells; angiogenesis; Notch signaling pathway; iPSC

\section{Introduction}

Congenital heart disease (CHD) represents almost one-third of all congenital diseases [1] and occurs in nearly 1\% of live births [2]. The dextro-transposition of the great arteries (d-TGA) is one of the most common and severe types of CHD $[1,3]$ with a prevalence of $20-33$ per 100,000 newborns and an incidence of $5-8 \%$ of all CHD [4,5]. While $\mathrm{d}$-TGA is most frequent in males (ratio $2: 1$ ), there is no correlation with any chromosomopathy [6].

d-TGA is generally reported with Heterotaxy syndrome, but the etiology and morphogenesis of TGA are still largely unknown. Nonetheless, several genes, such as Pitx2 and $T b x 2$, have been evaluated in animal models [3,7]. During heart development, the cardiac tube expands and begins a morphogenetic looping process that ultimately brings the posterior part of the tube to a rostral position, dorsal to the outflow tract (OFT) [8]. During mid-development, the OFT is remodeled into separate pulmonary and aortic arteries, which involves direct interactions between myocardium, endocardium and neural crest cells [9]. Subendocardial extracellular matrix (ECM) provides physical support and signaling regulation to cells and regulates tissue morphology during embryonic development [10]. The correct rotation of the myocardial wall of the OFT is necessary for the normal position of the great arteries. A high proportion of Perlecan (Hspg2)-null mouse embryos present with complete TGA [11], suggesting the importance of the ECM and 
basement membranes in cardiac morphogenesis. d-TGA can also be induced by retinoic acid treatments [12], directly involving the Tbx2-Tgf $\beta 2$ pathway [7].

Several studies have used animal models of TGA to better understand disease mechanisms, and there have only been a few studies reported in humans (reviewed in [3]). The emergence of human induced pluripotent stem cells (iPSC) [13] has raised the possibility of more robust disease modeling [14]. Due to the importance of endothelial cells (EC) in the vascular system, several studies have focused on designing robust protocols to differentiate iPSC into endothelial cells (iPSC-ECs) $[15,16]$.

In the present study, we sought to generate a d-TGA cellular model based on the use of patient-specific iPSCs. Specifically, we generated two iPSC lines from patients with d-TGA, which were differentiated into iPSC-ECs. Several genes were identified by RNA-seq as potential players in the etiology of this congenital defect. We show that iPSCECs from patients with d-TGA have an impaired capacity to develop vascular networks concomitant with changes in the transcriptome, as assessed by bioinformatics analysis. Specifically, gene ontology (GO) biological processes related to embryonic morphogenesis/development, tube development and angiogenesis, together with Notch signaling pathways, were downregulated in EC derived from patients with d-TGA.

\section{Results}

\subsection{Generation of Endothelial Cells from Differentiated iPSCs}

We generated iPSC lines from two patients with d-TGA (TGA1 and TGA2) and also one control iPSC line from a healthy individual (Ctrl1), which were cultured under feederfree conditions. We used the commercially available iPSC line IMR90-4 as a second control (Ctrl2). The cells were maintained undifferentiated and displayed typical morphology (Figure 1A) and normal karyotype (Figure S1). We differentiated the iPSC lines to ECs by the addition of different growth factors (Figure 1B), following a published protocol [15]. We then isolated cells that were double-positive for CD31 and neuropilin-1 (NRP-1), a coreceptor of vascular endothelial growth factor (VEGF), at day 12, using fluorescence-activated cell sorting (FACS), which ensured an EC population. The results of the sorting analysis revealed a similar but low efficiency of EC differentiation in all four iPSC lines: FACS efficiency was $0.93 \pm 0.69$ (Ctrl1), $2.8 \pm 2.08$ (Ctrl2), $0.57 \pm 0.23$ (TGA1) and $0.6 \pm 0.4$ (TGA2). Isolated ECs showed a characteristic cobblestone morphology by brightfield microscopy (Figure 1C) and were able to grow up to four passages. During endothelial differentiation of iPSC, we obtained RNA samples from each cell line at days 0, 5, 12 and 19 for gene characterization by qPCR. As expected, the levels of the pluripotency gene OCT4 decreased over time in all the lines tested. By contrast, the expression of the specific endothelial gene $C D 31$, vascular-endothelial cadherin (CDH5) and receptor tyrosine kinase (TIE2) increased over time (Figure 1D). All iPSC lines exhibited an increase in CD31 and TIE2 expression over time, indicating the differentiation of iPSC into EC. The expression profile of OCT4 and CD31 was similar in all the iPSC lines; however, some differences were observed when CDH5 and TIE2 were analyzed (Figure 1C).

\subsection{RNA-Seq Analysis of Endothelial Cells from Differentiated Control and d-TGA iPSC Lines}

We next performed RNA-seq and bioinformatics analysis of sorted double-positive cells at day 20 of differentiation of the four iPSC-EC lines. Despite the known variability that exists between individuals $[17,18]$ and the different origin of the iPSC lines used, the iPSC-EC samples from controls and patients with d-TGA were well separated by the principal component analysis (Figure 2A). These results support the notion that despite the interindividual genotypic differences, there were robust genetic differences between control and TGA lines. 
A

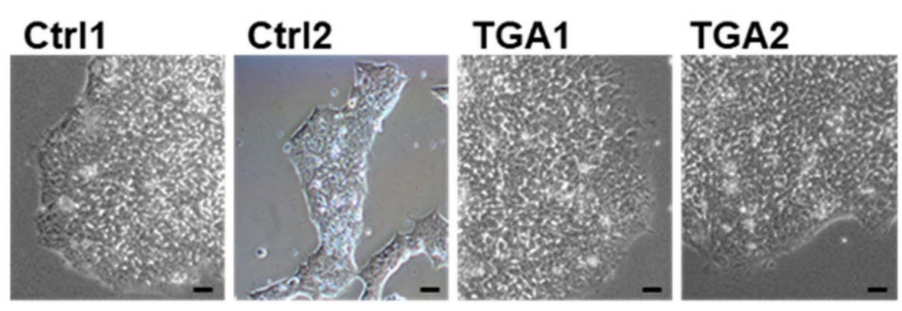

B

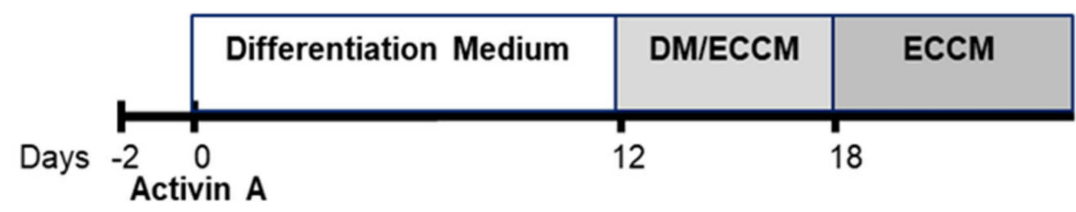

C
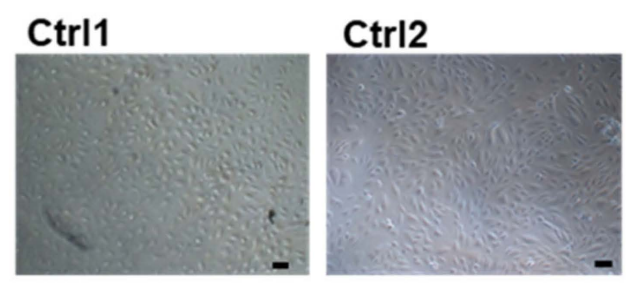

TGA1

TGA2

D
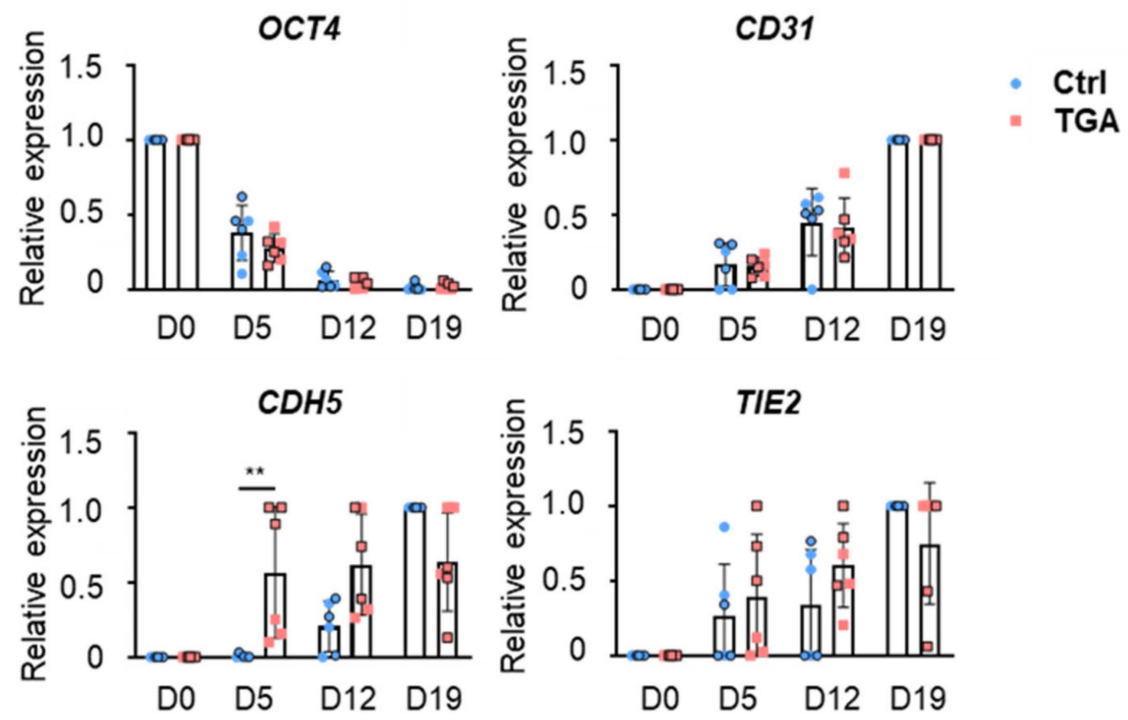

Figure 1. Differentiation of iPSC lines from control and d-TGA patients. (A) Representative brightfield images of undifferentiated iPSC colonies on Matrigel. (B) Protocol used for differentiation of iPSC lines to EC using differentiation medium (DM) and endothelial cell culture medium (ECCM). (C) Representative brightfield images of iPSC-ECs at day 19. Images were recorded using a $10 \times$ objective, scale bar $=10 \mu \mathrm{m}$. (D) Expression of genes involved in EC differentiation measured by qPCR in control (blue, $\bullet$ Ctrl1, $\bigcirc$ Ctrl2) and d-TGA lines (red, $\mathbf{a}$ TGA1, $\square$ TGA2). Samples were obtained at days 0, 5, 12 and 19 of EC differentiation. Data were normalized to GAPDH expression and are represented as mean \pm SD. Three independent differentiations were performed for each iPSC line. ${ }^{* *} p<0.01$, by Mann-Whitney U test. Scale bar $=10 \mu \mathrm{m}$. 
A

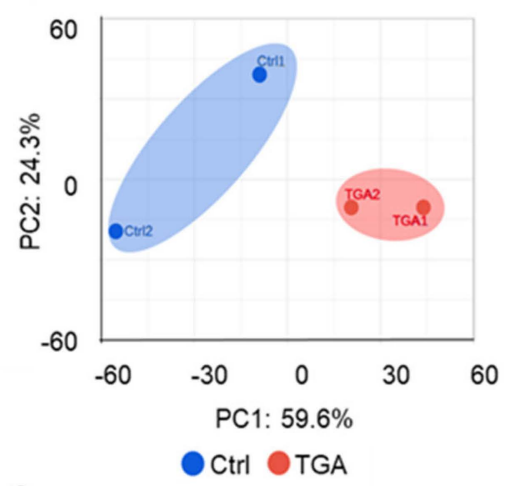

B

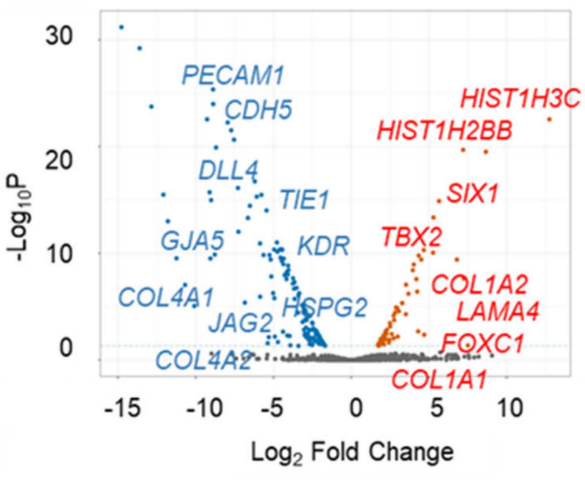

C

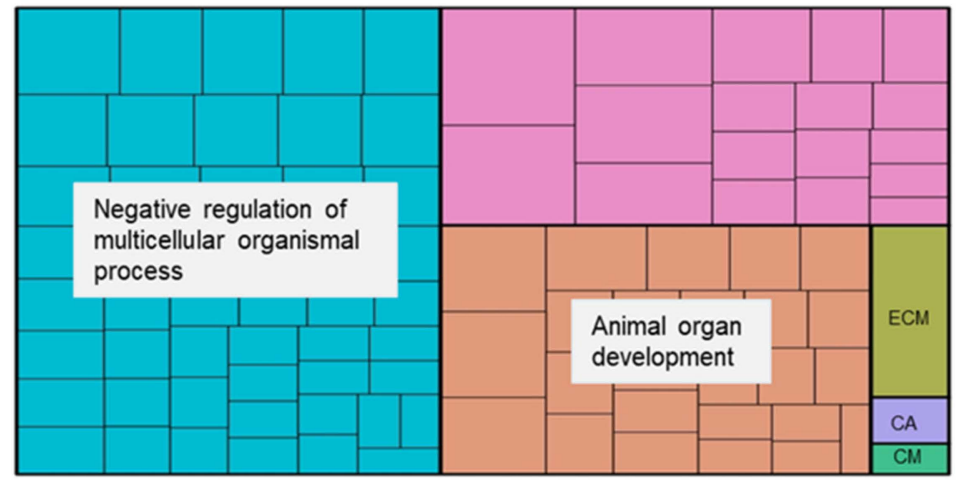

D

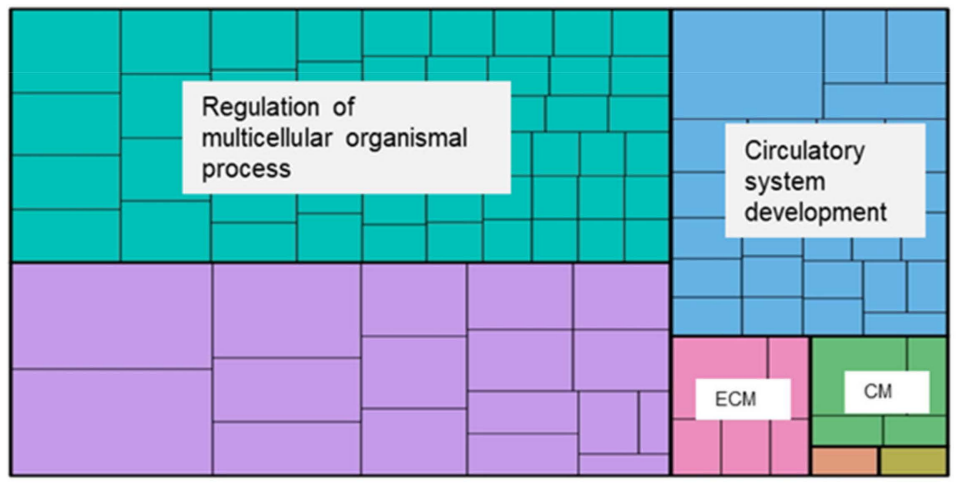

Figure 2. Transcriptome analysis of iPSC-ECs. (A) Principal component analysis of component 1 versus component 3 . (B) Volcano plot representing the distribution of log2 gene ratio between iPSC from d-TGA patients versus controls versus $\log 10$ gene intensity. Down- and upregulated genes are plotted in blue and red, respectively. (C) Treemap diagram of biological processes of upregulated genes overrepresented in iPSC-ECs from d-TGA versus control lines using REVIGO after g:Profiler gene analysis. (D) Treemap diagram of biological processes of downregulated genes, overrepresented in iPSC-ECs from d-TGA versus control lines using REVIGO after g:Profiler gene analysis. ECM: extracellular matrix organization, CM: cell motility and CA: cell adhesion. GO processes in non-labelled superclusters correspond to non-classified biological processes.

A total of 4672 genes were identified by bioinformatics analysis, and 4471 genes had similar expression values in d-TGA and control groups. Identified genes with fewer than 150 counts were removed. We generated a volcano plot to visualize differentially expressed genes (DEG) ( $p$-value $\leq 0.05$ and fold change $\geq 2.0$ ) in iPSC-ECs from d-TGA and control lines (Figure 2B). A total of 201 DEG were identified, of which 62 were upregulated in d-TGA lines and 139 were downregulated. Regarding the upregulated genes, we identified 
several genes associated with the extracellular matrix (ECM), such as collagen type I alpha 1 (COL1A1), COL1A2 and laminin subunit alpha 4 (LAMA4). We also found an increase in the expression of the transcription factors SIX1 and TBX2, which have important roles in heart morphogenesis $[8,19]$. A member of the Fox transcription factor family (FOXC1) with an important role in vascular development was also upregulated in iPSC-ECs from patients with d-TGA.

Of the 139 downregulated genes in the d-TGA group, we identified several genes involved in vascular compartment and developmental processes. For example, specific endothelial markers, including PECAM1, CDH5, TIE1 and VEGF receptor 2 (KDR) [20] were all downregulated in iPSC-ECs from patients with d-TGA, as well as genes involved in Notch signaling, including jagged canonical Notch ligand 2 (JAG2) and Delta-like canonical Notch ligand 4 (DLL4). In addition, genes involved in gap junctions, such as gap junction protein alpha 4 (GJA4) and GJA5, which are necessary to establish cell communication, were also downregulated. Likewise, vascular stabilizing genes related to the ECM, such as collagen type IV alpha 1 chain (COL4A1), COL4A2 and HSPG2, were downregulated. A complete list of the upregulated and downregulated DEG is shown in Supplementary Tables S1 and S2, respectively.

\section{3. iPSC-ECs from Patients with d-TGA Show Alterations in Signaling Pathways}

In agreement with the gene expression profiling, GO analysis of DEG demonstrated different biological processes associated with control and d-TGA iPSC-ECs. A list of all significantly overrepresented GO biological processes of upregulated and downregulated genes, as a function of their $p$-value, is shown in Supplementary Tables S3 and S4, respectively. Many of the GO processes identified in upregulated genes were involved with developmental processes, including animal organ development and morphogenesis, embryonic development and morphogenesis, negative regulation of developmental process and anatomical structure development (Table 1).

Table 1. Gene ontology biological processes associated with differentially upregulated genes in iPSC-ECs from patients with transposition of the great arteries versus healthy controls.

\begin{tabular}{ccc}
\hline GO_ID & Term & $p$-Value \\
\hline GO:0009887 & Animal organ morphogenesis & $1.59 \times 10^{-12}$ \\
GO:0048513 & Animal organ development & $1.84 \times 10^{-10}$ \\
GO:0043062 & Extracellular structure organization & $5.34 \times 10^{-9}$ \\
GO:0048856 & Anatomical structure development & $1.95 \times 10^{-6}$ \\
GO:0051093 & Negative regulation of developmental process & $3.13 \times 10^{-6}$ \\
GO:0009790 & Embryonic development & $9.05 \times 10^{-5}$ \\
GO:0045995 & Regulation of embryonic development & $1.10 \times 10^{-3}$ \\
GO:0072359 & Circulatory system development & $4.52 \times 10^{-3}$ \\
GO:0009792 & Embryonic development ending in birth or egg hatching & $6.98 \times 10^{-3}$ \\
GO:0060560 & Developmental growth involved in morphogenesis & $2.60 \times 10^{-2}$ \\
GO:0048568 & Embryonic organ development & $2.60 \times 10^{-2}$ \\
GO:0035148 & Tube formation & $3.10 \times 10^{-2}$ \\
GO:0048562 & Embryonic organ morphogenesis & $4.81 \times 10^{-2}$ \\
\hline
\end{tabular}

As a complementary representation, we used a REVIGO treemap to reduce the number of GO terms into 'clusters' and 'superclusters' of related terms, as described [21], where GO terms are visualized with different colors in rectangles representing the $p$-value (Figure 2C). The 304 GO biological processes identified were mainly grouped into two superclusters: 'negative regulation of multicellular organismal process', which was the supercluster most represented, and 'animal organ development'. We performed the same enrichment analysis on the downregulated genes; 279 GO terms were identified ( $p$-value $<0.05)$ and the most representative are listed in Table 2. Of note, many processes were related to blood vessel development and morphogenesis, tube development and morphogenesis, 
negative regulation of developmental process and regulation of blood vessel endothelial cell proliferation involved in sprouting angiogenesis.

Table 2. Gene ontology biological processes associated with differentially downregulated genes in iPSC-ECs from patients with transposition of the great arteries versus healthy controls.

\begin{tabular}{ccc}
\hline GO_ID & Term & $p$-Value \\
\hline GO:0072359 & Circulatory system development & $5.44 \times 10^{-15}$ \\
GO:0007275 & Multicellular organism development & $3.94 \times 10^{-12}$ \\
GO:0001568 & Blood vessel development & $6.28 \times 10^{-12}$ \\
GO:0048646 & Anatomical structure formation involved in morphogenesis & $7.67 \times 10^{-12}$ \\
GO:0048514 & Blood vessel morphogenesis & $7.67 \times 10^{-12}$ \\
GO:0072358 & Cardiovascular system development & $7.67 \times 10^{-12}$ \\
GO:0048856 & Anatomical structure development & $2.86 \times 10^{-10}$ \\
GO:0035239 & Tube morphogenesis & $3.65 \times 10^{-10}$ \\
GO:0032502 & Developmental process & $4.03 \times 10^{-10}$ \\
GO:0035295 & Tube development & $1.07 \times 10^{-9}$ \\
GO:2000181 & Negative regulation of blood vessel morphogenesis & $1.09 \times 10^{-5}$ \\
GO:0022603 & Regulation of anatomical structure morphogenesis & $1.10 \times 10^{-5}$ \\
GO:0007507 & Heart development & $1.42 \times 10^{-5}$ \\
GO:0050793 & Regulation of developmental process & $1.37 \times 10^{-4}$ \\
GO:0051093 & Negative regulation of developmental process & $1.10 \times 10^{-3}$ \\
GO:0003230 & Cardiac atrium development & $5.46 \times 10^{-3}$ \\
GO:1903587 & Regulation of blood vessel endothelial cell proliferation & $1.48 \times 10^{-2}$ \\
\hline
\end{tabular}

These results suggest that the downregulated genes identified in iPSC-ECs from patients with d-TGA are of particular relevance for understanding the mechanisms of d-TGA in our system.

We also created a treemap to group the GO biological processes, which were mostly grouped into the 'regulation of multicellular organismal process' and 'circulatory system development' superclusters (Figure 2D).

Several GO biological processes were identified when the analysis was performed with up- and downregulated genes. GO analysis of genes downregulated in iPSC-ECs from patients with d-TGA versus controls revealed significant enrichment of genes involved in the circulatory system.

We next conducted an interactome analysis to study the interaction network between the targets of DEG in iPSC-ECs from patients with d-TGA versus controls (Figure 3). GO biological processes were identified with gene-set enrichment analysis, and the interaction network was visualized with Cytoscape software v3.7.2.

When targets of upregulated genes were analyzed, biological processes related to anatomical structure morphogenesis and development, regulation of the developmental process, embryonic morphogenesis and development were enriched in iPSC-ECs of patients with d-TGA (Table 3).

When targets of downregulated genes were analyzed, biological processes related to cellular and animal organ development processes were enriched in iPSC-ECs of patients with d-TGA (Table 4). In agreement with the aforementioned results, processes related to circulatory system development, blood vessel development and morphogenesis, and Notch signaling were also identified. 


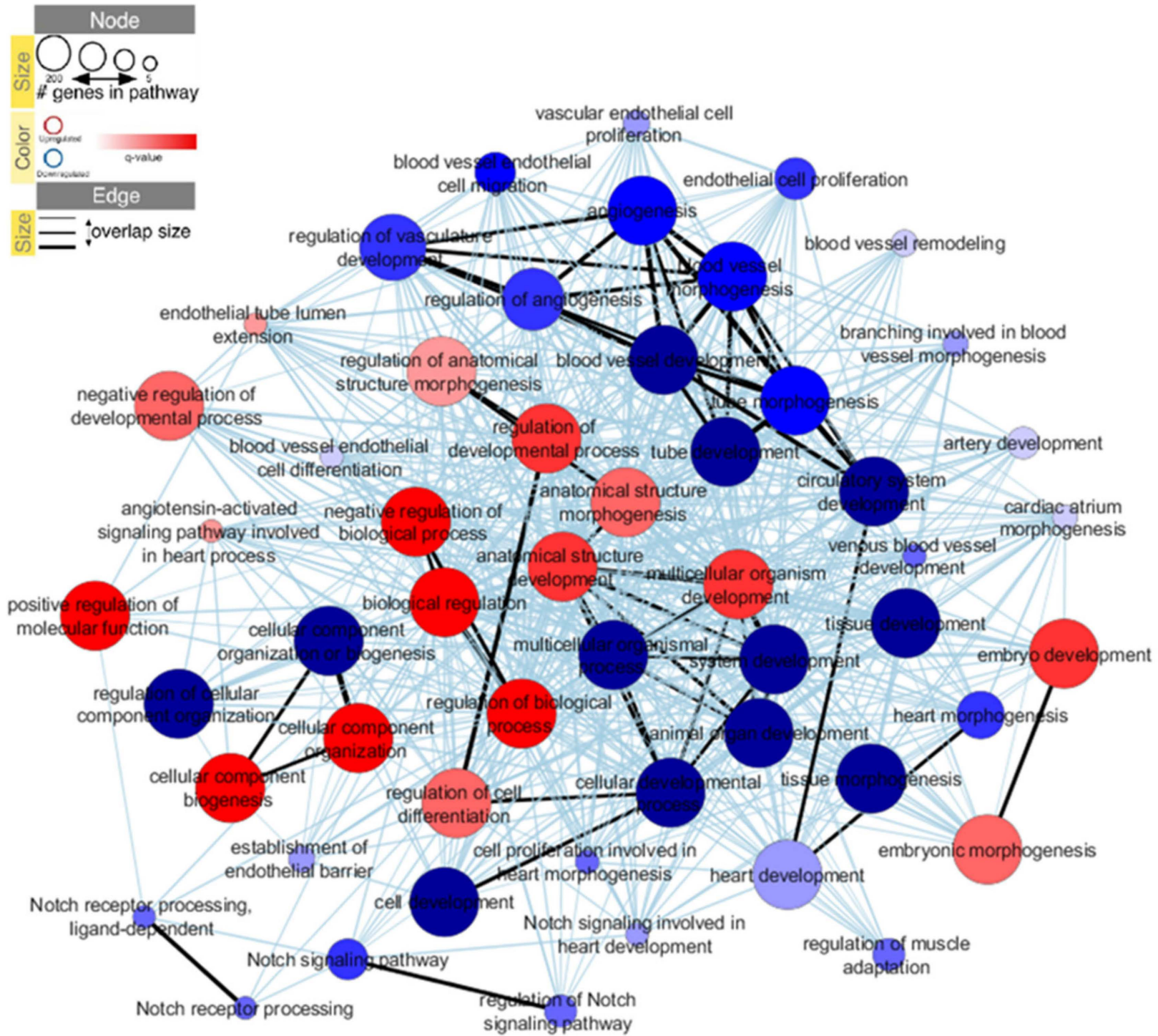

Figure 3. Graphical representation of the iPSC-EC interaction network based on RNA-seq data sets. Biological processes are represented as dots, where the size depends on the number of genes identified in each biological process. Upregulated and downregulated biological processes are represented in red and blue, respectively. The size of the nodes is represented as a function of the number of genes involved in each of the pathways and the colors as a function of the $p$-value. Stronger associations are now represented with black lines, while the others are colored in light blue.

Table 3. Gene ontology biological processes identified from the interactome analysis of upregulated genes in iPSC-ECs from patients with transposition of the great arteries versus healthy controls.

\begin{tabular}{lcc}
\hline GO_ID & Term & $p$-Value \\
\hline GO:0050789 & Regulation of biological process & $1.39 \times 10^{-11}$ \\
GO:0050793 & Regulation of developmental process & $7.00 \times 10^{-9}$ \\
GO:0007275 & Multicellular organism development & $3.40 \times 10^{-8}$ \\
GO:0048856 & Anatomical structure development & $8.06 \times 10^{-8}$ \\
GO:0048513 & Animal organ development & $3.38 \times 10^{-7}$ \\
GO:0009790 & Embryonic development & $8.96 \times 10^{-7}$ \\
GO:0009653 & Anatomical structure morphogenesis & $6.32 \times 10^{-5}$ \\
GO:0048598 & Embryonic morphogenesis & $4.82 \times 10^{-3}$ \\
GO:0051093 & Negative regulation of developmental process & $1.50 \times 10^{-2}$ \\
GO:0086098 & Angiotensin-activated signaling pathway involved in heart process & $3.62 \times 10^{-2}$ \\
\hline
\end{tabular}


Table 4. Gene ontology biological processes identified from the interactome analysis of downregulated genes in iPSC-ECs from patients with transposition of the great arteries versus healthy controls.

\begin{tabular}{ccc}
\hline GO_ID & Term & $p$-Value \\
\hline GO:0051128 & Regulation of cellular component organization & $1.91 \times 10^{-31}$ \\
GO:0048869 & Cellular developmental process & $6.91 \times 10^{-23}$ \\
GO:0048731 & System development & $3.88 \times 10^{-22}$ \\
GO:0009888 & Tissue development & $1.99 \times 10^{-19}$ \\
GO:0048513 & Animal organ development & $4.43 \times 10^{-15}$ \\
GO:0032501 & Multicellular organismal process & $3.89 \times 10^{-13}$ \\
GO:0048729 & Tissue morphogenesis & $5.28 \times 10^{-12}$ \\
GO:0072359 & Circulatory system development & $8.95 \times 10^{-9}$ \\
GO:0001568 & Blood vessel development & $3.60 \times 10^{-8}$ \\
GO:0035295 & Tube development & $9.09 \times 10^{-8}$ \\
GO:0048514 & Blood vessel morphogenesis & $9.56 \times 10^{-8}$ \\
GO:0001525 & Angiogenesis & $1.35 \times 10^{-7}$ \\
GO:0035239 & Tube morphogenesis & $2.12 \times 10^{-7}$ \\
GO:0007219 & Notch signaling pathway & $7.34 \times 10^{-6}$ \\
GO:0045765 & Regulation of angiogenesis & $9.75 \times 10^{-6}$ \\
GO:1901342 & Regulation of vasculature development & $1.80 \times 10^{-5}$ \\
GO:0009790 & Embryonic development & $6.51 \times 10^{-5}$ \\
GO:0003007 & Heart morphogenesis & $7.34 \times 10^{-4}$ \\
GO:0007220 & Notch receptor processing & $2.93 \times 10^{-3}$ \\
GO:0035333 & Notch receptor processing, ligand-dependent & $5.73 \times 10^{-3}$ \\
GO:0060841 & Venous blood vessel development & $5.95 \times 10^{-3}$ \\
GO:0061028 & Notch signaling involved in heart development & $1.06 \times 10^{-2}$ \\
GO:0060837 & Blood vessel endothelial cell differentiation & $2.16 \times 10^{-2}$ \\
GO:0060840 & Artery development & $2.88 \times 10^{-2}$ \\
GO:0001974 & Blood vessel remodeling & $3.36 \times 10^{-2}$ \\
\hline
\end{tabular}

2.4. Capillary-like Tube Formation Processes Are Altered in iPSC-ECs from Patients with d-TGA

The results of the bioinformatics analysis suggested that iPSC-ECs from patients with d-TGA might show defective blood vessel development and morphogenesis. To test this, we performed a functional in vitro assay with iPSC-ECs to evaluate their capacity to form a capillary-like tube network, as a readout of angiogenesis. Cells were seeded onto Matrigel in EGM-2 medium, and tube formation was evaluated microscopically after $3 \mathrm{~h}$. Results showed that iPSC-ECs from patients with d-TGA were unable to correctly form tubular structures measured as total loops, total tube length, number of total tubes and branching points (Figure $4 \mathrm{~A}, \mathrm{~B}$ ), with significant differences in other parameters, including covered area (\%), mean loop area and mean loop perimeter (Figure 4B). By contrast, the total net abundance was significantly higher in iPSC-ECs from patients with d-TGA, and no differences were found when the mean tube length was measured. These results are in line with the interactome analysis, where biological processes related to the regulation of angiogenesis were downregulated (Figure 3). 
A
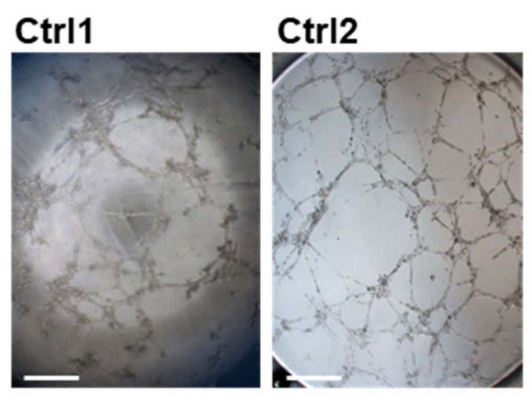

TGA1

TGA2
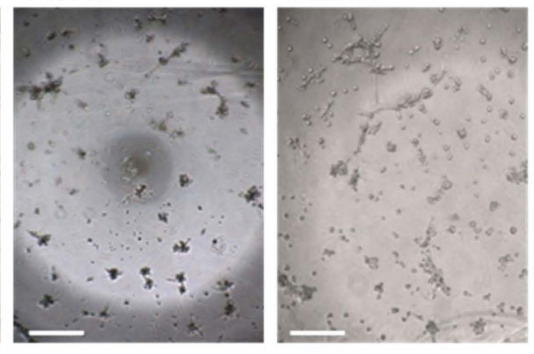

B
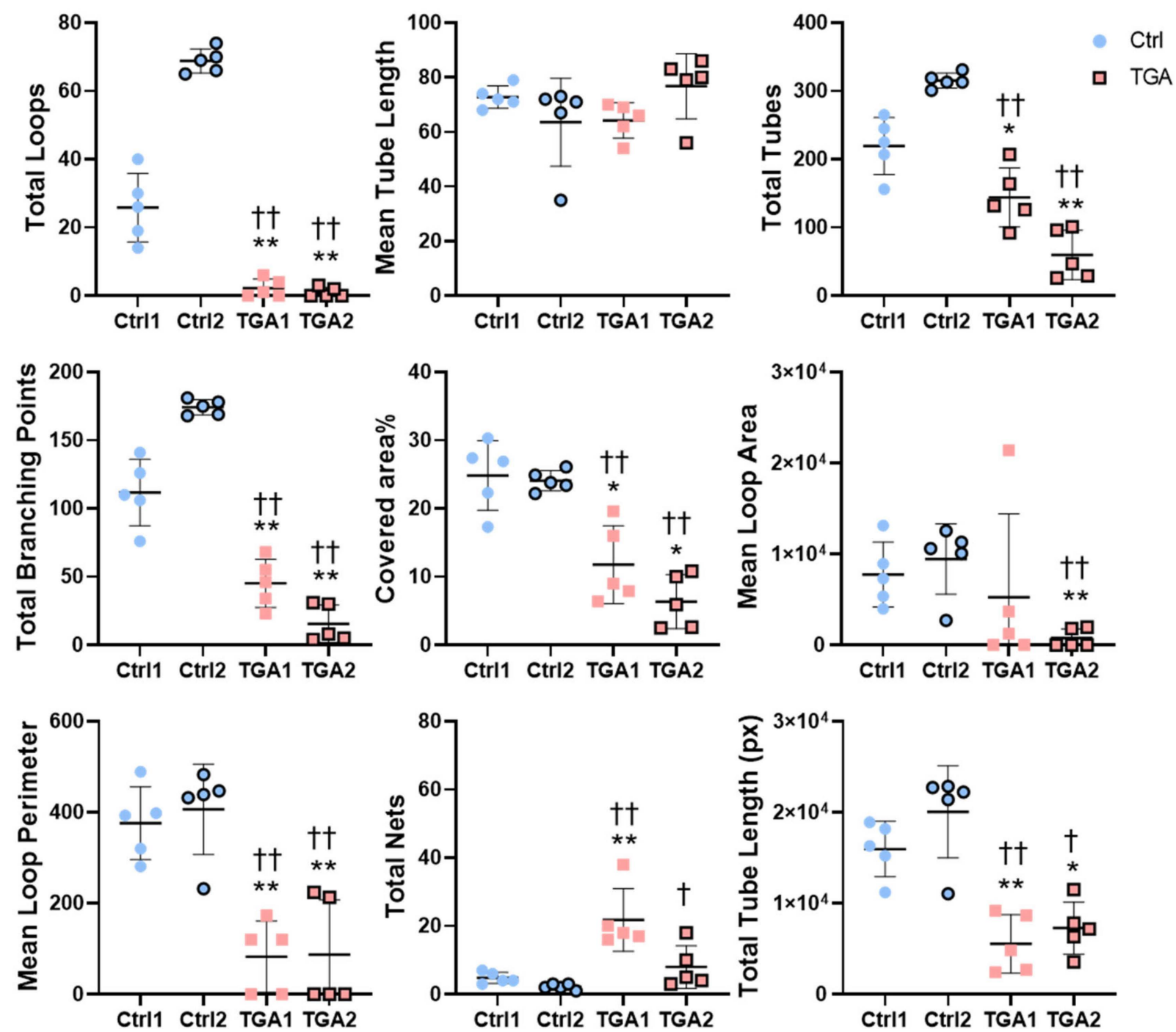

- Ctrl1 $\circ$ Ctrl2 - TGA1 $\square$ TGA2

Figure 4. Functional analysis of iPSC-ECs. (A) Representative images of tube formation assay $3 \mathrm{~h}$ after seeding iPSC-ECs from control and d-TGA lines. (B) Quantification of total loops, total mean tube length, total tubes, total branching points, covered area (\%), mean loop area and mean loop perimeter, total nets and mean tube length from images in control (blue, - Ctr11, $\bigcirc$ Ctrl2) and d-TGA (red, $\square$ TGA1, $\square$ TGA2) lines. Data are represented as mean \pm SD, results are expressed in arbitrary units $\left(n=5,{ }^{*} p<0.05,{ }^{* *} p<0.01\right.$ by Mann-Whitney $\mathrm{U}$ test in Ctrl1 versus TGA1 or TGA2 and $+p<0.05,+\dagger p<0.01$ in Ctrl2 versus TGA1 or TGA2). 


\section{Discussion}

d-TGA is the sixth most prevalent CHD from a total of 27 anatomical subtypes, as reported in a systematic review [22]. The advent of human iPSC technologies in 2007 by Yamanaka et al. [13] has been a major breakthrough for disease modeling and for therapeutic purposes [14], and detailed gene analysis of iPSC lines from patients with $\mathrm{CHD}$ is a promising strategy to study the mechanisms involved in the onset of cardiac diseases [23].

We studied the gene expression patterns in iPSC-ECs from patients with d-TGA, the deregulation of which may be related to the onset of disease. Two iPSC lines were successfully generated from patients with d-TGA and were differentiated into ECs. Despite the low efficiency of EC differentiation, the FACS-sorted CD31+/NRP-1+ double-positive cells proliferated and expressed endothelial markers. We used bioinformatics analysis to identify genes differentially expressed in iPSC-ECs from patients with d-TGA. One of these genes was the transcription factor $T b x 2$, which was overexpressed in ECs from patients with d-TGA. Tbx2 expression is closely related to heart development, as it is expressed in the heart tube, and it has been described to arrest cardiac development at looping [8]. It has also been observed to be highly expressed in the early stages of development, but it decreases over time [24]. Indeed, Tbx2 expression is involved in other aspects of heart looping but is not related to chamber growth [25]. Another gene upregulated in iPSC-ECs from patients with d-TGA was FOXC1, which has been described to promote angiogenic activity in vascular endothelial cells that is determined by the correct balance between pro-angiogenic FOXC1 activity in the endothelium and anti-angiogenic FOXC1 activity in the surrounding cells [26]. Two members of the histone family, HIST1H3C and HIST1H2BB, were also upregulated in iPSC-ECs from d-TGA patients. Altered expression of different histones has been related to vascular diseases, such as abdominal aortic aneurysm, which is characterized by the degradation of the elastic media and remodeling of the aortic ECM [27]. Different genes upregulated in iPSC-ECs from d-TGA (LAMA4, COL1A1, COL1A2 and COL6A1) are involved in ECM organization (GO:0030198). These data suggest that the expression of different genes related to ECM is altered in iPSC-ECs from patients with d-TGA. HSPG2, a glycosylated protein component of the ECM, was downregulated in iPSCECs from d-TGA patients. Interestingly, Hspg2-null embryos presented with d-TGA [11], supporting the important role of this gene and genes related to ECM in the development of the disease.

Genetic polymorphisms in GJA4 have been previously related to coronary artery disease [28]. This gene was downregulated in iPSC-ECs from d-TGA patients, suggesting that both Tbx2 and GJA4 might be implicated in the development of d-TGA.

Regarding the downregulated genes expressed in EC from patients with d-TGA, we found several different genes involved in Notch signaling. One of these genes, JAG2, is directly implicated in angiogenesis [29], and DLL4 is required for normal arterial patterning [30]. In addition, DLL4 has an antiangiogenic effect when it competes with JAG1 [31]. Thus, different genes involved in Notch signaling could be directly involved in disease development. CDH5 was also found to be downregulated in iPSC-ECs from patients with d-TGA. CDH5 is a transmembrane cadherin protein required for vascular morphogenesis, as its deficiency impairs vasculogenesis in embryonic stem cells derived from embryoid bodies [32] and in mutant mice embryos [33]. These defects were observed, even though the endothelial cells were well differentiated [33]. The downregulation of $K D R$, which has an important role in endothelial specification and maintenance, was also observed. The activation of this receptor is critical for enhancing the proliferation and survival potential of iPSC-ECs [15].

At the functional level, we observed that endothelial cells derived from patients with d-TGA were unable to form capillary-like tubular networks. ECs are directly implicated in angiogenesis, which plays a crucial role during embryonic development and organogenesis [34]. These findings might be related to the low expression levels of genes involved in the Notch signaling pathway observed in the bioinformatics analysis. 
The major limitation of our study is the lack of a multicellular system to simulate d-TGA in vitro. However, we have generated an in vitro model of d-TGA using iPSCs as a disease modeling approach. The gene expression differences between the ECs from control and d-TGA patients might be related to the etiology of this cardiac disease. The results presented in this work contribute to a better understanding of the mechanisms promoting the onset of d-TGA during embryonic development.

Overall, our results show that ECs from patients with d-TGA display genetic differences versus control counterparts. These changes affect biological processes related to the circulatory system and to vascular development. Accordingly, ECs derived from d-TGA iPSC s could be a good cellular model for the study of this pathology. This work supports the use of patient-specific iPSC-ECs in modeling d-TGA.

\section{Materials and Methods}

\subsection{Human-Induced Pluripotent Cell Generation}

Foreskin fibroblasts from a healthy individual and a patient with d-TGA (Ctrl1 and TGA1) were obtained through ATCC and the Coriell Institute, respectively. Fibroblasts were reprogramed following the method described by Yu in 2007 [35], using a lentiviral vector expressing the Oct4, Nanog, Lin 28 and Sox2 genes. Different clones were obtained and were validated using the following assays: alkaline phosphatase, surface marker expression, pluripotency gene expression, transgene silencing, proviral integration and in vitro and in vivo differentiation (data not shown).

Ctrl2 line (IMR90-4) was purchased from WiCell Research Institute (Madison, WI, USA) and was reprogramed using viral transfection (Oct4, Sox2, Nanog and Lin28). The iPSCTGA line TGA2 (SCVI-235) was generated from peripheral blood mononuclear cells [36] and was obtained from the Stanford Cardiovascular Institute iPSC Biobank (Stanford, CA, USA).

\subsection{Cell Culture}

All pluripotent cell lines and differentiation cultures were maintained in Matrigel (Corning, Bedford, MA, USA) pre-coated plates at $37^{\circ} \mathrm{C}$ in a Thermo Forma 370 Steri Cycle Incubator (Thermo Fisher Scientific, Walthman, MA, USA) with 5\% $\mathrm{CO}_{2}$ and $21 \% \mathrm{O}_{2}(21 \%$ $\mathrm{O}_{2}$ ). Cells were grown with mTeSR1 medium (Stemcell Technologies, Vancouver, Canada) and were passaged at a 1:10 ratio when $\sim 80 \%$ confluency was reached (after about 4 days) using Accutase solution (Stemcell Technologies).

\subsection{Endothelial Differentiation}

Endothelial cell differentiation was induced following the protocol described by Prasain et al. in 2014 [15]. Briefly, cells were seeded at a density of $6250 \mathrm{~cm}^{-2}$ onto a Matrigel pre-coated 24-well plate (day-2) and were incubated for 2 days in mTeSR1 medium. On day 0, cells were cultured with Stemline ${ }^{\circledR}$ II Hematopoietic Stem Cell Expansion Medium (Sigma-Aldrich, Darmstadt, Germany) containing $10 \mathrm{ng} / \mathrm{mL}$ of Activin A (R\&D Systems, Minneapolis, MN, USA), FGF-2 (Tebu-bio, Yvelines, France), BMP-4 (R\&D Systems, Minneapolis, MN, USA) and VEGF (Peprotech, London, UK) for 1 day to initiate the differentiation. The following day, the medium was replaced and cells were incubated with the same concentration of FGF-2, BMP-4 and VEGF (differentiation medium) for 11 days. On day 12, FACS was performed to isolate endothelial cells.

\subsection{Fluorescence-Activated Cell Sorting}

Cells were detached by trypsinization and filtered through a $40-\mu \mathrm{m}$ filter to form a single cell suspension. Cells were resuspended in $130 \mu \mathrm{L}$ of PBS, supplemented with $1 \%$ fetal bovine serum and were incubated with anti-human platelet/endothelial cell adhesion molecule-1 (CD31; PECAM-1, Alexa Fluor 488-conjugated, BD-Pharmingen, San Diego, CA, USA) and Neuropilin-1 (NRP-1, APC-conjugated, Miltenyi Biotech, Bergisch Gladbach, Germany). Double-positive CD31+/NRP-1+ cells were isolated by FACS and 
were expanded in collagen-coated T25 flasks until confluence [37] in Endothelial Cell Growth Medium-2 (EC medium) BulletKit ${ }^{\mathrm{TM}}$ (Lonza, Basel, Switzerland). Experiments were performed from day 19 of differentiation and cells were maintained in culture for no more than 5 passages.

\subsection{Tube Formation Assay}

iPSC-ECs were plated onto Matrigel-coated wells to measure tube formation, as described [38]. Briefly, $20 \times 10^{4}$ cells were seeded per well into 96-well plates and were incubated for $3 \mathrm{~h}$. Images were acquired using an inverted microscope (Leica DM6000, Leica Microsystems, Wetzlar, Germany) with a $10 \times$ magnification and were analyzed with WimTube online software (WimTube: Tube Formation Assay Image Analysis. Release 4.0. https:/ / www.wimasis.com/en/WimTube, accessed on 06 December 2021 ). Five images from independent experiments were analyzed for each iPSC-EC line.

\subsection{RNA Extraction and Quantitative Real-Time PCR}

RNA was isolated from samples on different days of EC differentiation (days 0, 5, 12 and 19) using the RNeasy Plus kit (Qiagen, Dusseldorf, Germany). cDNA was produced using a High-Capacity RNA-to-cDNA Kit (Applied Biosystems, Walthman, MA, USA), and real-time PCR (qPCR) was performed using the LightCycler 480 SYBR Green I Master Kit (Roche Life Science, Switzerland) on a ViiA 7 Real-Time PCR System (Applied Biosystems, Walthman, MA, USA). Primers were provided by Condalab (Madrid, Spain) and glyceraldehyde-3-phosphate dehydrogenase $(G A P D H)$ was used as housekeeping control. Reactions were performed in triplicate.

\subsection{RNA-Seq}

Libraries were prepared using the TruSeq Stranded Total RNA Library Prep Kit with Ribo-Zero Human/Mouse/Rat Kit (Illumina, San Diego, CA, USA). Briefly, 300 ng of total RNA was used for ribosomal RNA depletion. Then, ribosomal-depleted RNA was fragmented for $4.5 \mathrm{~min}$ at $94{ }^{\circ} \mathrm{C}$. The remaining steps of the library preparation were followed according to the manufacturer's instructions.

Libraries were analyzed on an Agilent Technologies 2100 Bioanalyzer system, using the Agilent DNA 1000 chip to estimate the quantity and validate the size distribution, and were then quantified by qPCR using the KAPA Library Quantification Kit KK4835 (Roche Life Science, Switzerland).

FASTQ files were treated with Cutadapt and aligned with Kallisto to obtain the count table for each gene. Differential expression analysis of RNA-seq expression profiles was performed with the EdgeR package [39]. GO enrichment analyses were performed using g:Profiler [40,41].

\subsection{Interactome Analysis}

Analysis was performed as described [21]. Complete interactome was identified, and GSEA was performed to detect significant GO biological processes, molecular functions and cellular components [40].

\subsection{Statistical Analysis}

Results are represented as the mean \pm standard deviation (SD). Comparisons between Ctrl and TGA groups were performed with the non-parametric Mann-Whitney U test, one-way ANOVA and necessary post hoc analysis. Analyses were conducted with GraphPad Prism $8^{\circledR}$ software (GraphPad Software Inc., La Jolla, CA, USA). Differences were considered statistically significant at $p<0.05$, with a $95 \%$ confidence interval.

Supplementary Materials: The following are available online at https:/ / www.mdpi.com/article/10 $.3390 /$ ijms222413270/s1. 
Author Contributions: I.O.-O. and G.F. were responsible for design, performance, analysis, interpretation of the experiments and the manuscript writing; J.C.W. and T.K. were responsible for iPSC generation and review; S.T. was responsible for cell culture and supporting in vitro experiments; J.P. carried out bioinformatics studies; A.V. reviewed the manuscript; S.E.H. supervised the project, provided funding and reviewed the manuscript; P.S. was responsible for conceptualization, data curation, funding acquisition and review. All authors have read and agreed to the published version of the manuscript.

Funding: This work was supported by RETICS Program (RD16/0011/0004) from Instituto de Salud Carlos III, co-funded by FEDER 'una manera de hacer Europa'. This work was supported by the National Institutes of Health R01 HL130020, R01 HL145676 and R01 HL141851 to J.C.W. This work was supported by the BHF Centre grant RM/17/1/33377 to S.E.H. This work was also supported by a postdoctoral grant to I.O.-O. from Instituto de Investigación Sanitaria La Fe (2014/0151) and a predoctoral grant to S.T ACIF/2018/259 from the Conselleria de Sanitat Universal i Salut Pública and co-financed by the European Union through the Operational Programme European Regional Development Fund (FEDER) of the Valencian Community 2014-2020. G.F. was supported by Medical Research Council [MR/R025002/1], NIHR Imperial Biomedical Research Centre and the Hungarian National Research, Development and Innovation Fund [2020-1.1.6-JÖVÖ-2021-00013 and K128369].

Institutional Review Board Statement: The study was conducted according to the guidelines of the Declaration of Helsinki and approved by the Institutional Ethics Committee of Hospital Universitari i Politècnic La Fe (protocol code 2014/0151, date of approval 3 March 2016).

Informed Consent Statement: Written informed consent was obtained from all subjects involved in the study.

Data Availability Statement: The data presented in the study are deposited in the GEO database repository. The accession number is GEO:GSE186803.

Acknowledgments: The authors would like to thank the Genomics Unit at the CRG for assistance with the total RNA-seq. I.O-O acknowledges a postdoctoral contract from the PlaGenT, Generalitat Valenciana (CDPT-01/20-A). The authors thank the microscopy and cell culture core facilities of IIS La Fe and the Flow Cytometry Core Facility at Imperial College. We thank Sebastian-Leon for her help with the sequencing data analysis. We thank Kenneth McCreath for his constructive comments on the manuscript.

Conflicts of Interest: The authors declare that the research was conducted in the absence of any commercial or financial relationships that could be construed as a potential conflict of interest.

\section{References}

1. Van Der Linde, D.; Konings, E.E.M.; Slager, M.A.; Witsenburg, M.; Helbing, W.A.; Takkenberg, J.J.M.; Roos-Hesselink, J.W. Birth prevalence of congenital heart disease worldwide: A systematic review and meta-analysis. J. Am. Coll. Cardiol. 2011, 58, 2241-2247. [CrossRef] [PubMed]

2. Kodo, K.; Nishizawa, T.; Furutani, M.; Arai, S.; Yamamura, E.; Joo, K.; Takahashi, T.; Matsuoka, R.; Yamagishi, H. GATA6 mutations cause human cardiac outflow tract defects by disrupting semaphorin-plexin signaling. Proc. Natl. Acad. Sci. USA 2009, 106, 13933-13938. [CrossRef] [PubMed]

3. Unolt, M.; Putotto, C.; Silvestri, L.M.; Marino, D.; Scarabotti, A.; Massaccesi, V.; Caiaro, A.; Versacci, P.; Marino, B. Transposition of great arteries: New insights into the pathogenesis. Front. Pediatr. 2013, 1, 11. [CrossRef] [PubMed]

4. Gutgesell, H.P.; Garson, A.; McNamara, D.G. Prognosis for the newborn with transposition of the great arteries. Am. J. Cardiol. 1979, 44, 96-100. [CrossRef]

5. Brickner, M.E.; Hillis, L.D.; Lange, R.A. Congenital heart disease in adults (Second of two parts). N. Engl. J. Med. 2000, 342, 334-342. [CrossRef]

6. Mégarbané, A.; Salem, N.; Stephan, E.; Ashoush, R.; Lenoir, D.; Delague, V.; Kassab, R.; Loiselet, J.; Bouvagnet, P. X-linked transposition of the great arteries and incomplete penetrance among males with a nonsense mutation in ZIC3. Eur. J. Hum. Genet. 2000, 8, 704-708. [CrossRef] [PubMed]

7. Sakabe, M.; Kokubo, H.; Nakajima, Y.; Saga, Y. Ectopic retinoic acid signaling affects outflow tract cushion development through suppression of the myocardial Tbx2-Tgf 2 pathway. Development 2012, 139, 385-395. [CrossRef] [PubMed]

8. Harrelson, Z.; Kelly, R.G.; Goldin, S.N.; Gibson-Brown, J.J.; Bollag, R.J.; Silver, L.M.; Papaioannou, V.E. Tbx2 is essential for patterning the atrioventricular canal and for morphogenensis of the outflow tract during heart development. Development 2004, 131, 5041-5052. [CrossRef]

9. Bruneau, B.G. The developmental genetics of congenital heart disease. Nature 2008, 451, 943-948. [CrossRef] 
10. Walma, D.A.C.; Yamada, K.M. The extracellular matrix in development. Development 2020, 147, dev175596. [CrossRef] [PubMed]

11. Costell, M.; Carmona, R.; Gustafsson, E.; González-Iriarte, M.; Fässler, R.; Muñoz-Chápuli, R. Hyperplastic Conotruncal Endocardial Cushions and Transposition of Great Arteries in Perlecan-Null Mice. Circ. Res. 2002, 91, 158-164. [CrossRef] [PubMed]

12. Yasui, H.; Nakazawa, M.; Morishima, M.; Miyagawa-Tomita, S.; Momma, K. Morphological observations on the pathogenetic process of transposition of the great arteries induced by retinoic acid in mice. Circulation 1995, 91, 2478-2486. [CrossRef] [PubMed]

13. Takahashi, K.; Tanabe, K.; Ohnuki, M.; Narita, M.; Ichisaka, T.; Tomoda, K.; Yamanaka, S. Induction of Pluripotent Stem Cells from Adult Human Fibroblasts by Defined Factors. Cell 2007, 131, 861-872. [CrossRef] [PubMed]

14. Choi, K.-D.; Yu, J.; Smuga-Otto, K.; Salvagiotto, G.; Rehrauer, W.; Vodyanik, M.; Thomson, J.; Slukvin, I. Hematopoietic and Endothelial Differentiation of Human Induced Pluripotent Stem Cells. Stem Cells 2009, 27, 559-567. [CrossRef]

15. Prasain, N.; Lee, M.R.; Vemula, S.; Meador, J.L.; Yoshimoto, M.; Ferkowicz, M.J.; Fett, A.; Gupta, M.; Rapp, B.M.; Saadatzadeh, M.R.; et al. Differentiation of human pluripotent stem cells to cells similar to cord-blood endothelial colony-forming cells. Nat. Biotechnol. 2014, 32, 1151-1157. [CrossRef] [PubMed]

16. James, D.; Nam, H.S.; Seandel, M.; Nolan, D.; Janovitz, T.; Tomishima, M.; Studer, L.; Lee, G.; Lyden, D.; Benezra, R.; et al. Expansion and maintenance of human embryonic stem cell-derived endothelial cells by TGFB inhibition is Id1 dependent. Nat. Biotechnol. 2010, 28, 161-166. [CrossRef] [PubMed]

17. Musunuru, K. Genome editing of human pluripotent stem cells to generate human cellular disease models. DMM Dis. Model. Mech. 2013, 6, 896-904. [CrossRef] [PubMed]

18. Carcamo-Orive, I.; Hoffman, G.E.; Cundiff, P.; Beckmann, N.D.; D’Souza, S.L.; Knowles, J.W.; Patel, A.; Papatsenko, D.; Abbasi, F.; Reaven, G.M.; et al. Analysis of Transcriptional Variability in a Large Human iPSC Library Reveals Genetic and Non-genetic Determinants of Heterogeneity. Cell Stem Cell 2017, 20, 518-532. [CrossRef]

19. Li, B.; Xu, L.; Hong, N.; Chen, S.; Xu, R. In Silico Analyses Reveal the Relationship Between SIX1/EYA1 Mutations and Conotruncal Heart Defects. Pediatr. Cardiol. 2018, 39, 176-182. [CrossRef] [PubMed]

20. Garlanda, C.; Dejana, E. Heterogeneity of endothelial cells: Specific markers. Arterioscler. Thromb. Vasc. Biol. 1997, 17, 1193-1202. [CrossRef] [PubMed]

21. Ontoria-Oviedo, I.; Palacios, I.; Panadero, J.; Sánchez, B.; García-García, F.; López-Cerdán, A.; Dorronsoro, A.; Castellano, D.; Rodríguez-Borlado, L.; Bernad, A.; et al. Plasmatic Membrane Expression of Adhesion Molecules in Human Cardiac Progenitor/Stem Cells Might Explain Their Superior Cell Engraftment after Cell Transplantation. Stem Cells Int. 2020, 2020, 8872009. [CrossRef] [PubMed]

22. Liu, Y.; Chen, S.; Zühlke, L.; Black, G.C.; Choy, M.K.; Li, N.; Keavney, B.D. Global birth prevalence of congenital heart defects 1970-2017: Updated systematic review and meta-analysis of 260 studies. Int. J. Epidemiol. 2019, 48, 455-463. [CrossRef] [PubMed]

23. Kitani, T.; Tian, L.; Zhang, T.; Itzhaki, I.; Zhang, J.Z.; Ma, N.; Liu, C.; Rhee, J.W.; Romfh, A.W.; Lui, G.K.; et al. RNA sequencing analysis of induced pluripotent stem cell-derived cardiomyocytes from congenital heart disease patients. Circ. Res. 2020, 126, 923-925. [CrossRef]

24. Christoffels, V.M.; Hoogaars, W.M.H.; Tessari, A.; Clout, D.E.W.; Moorman, A.F.M.; Campione, M. T-Box Transcription Factor Tbx2 Represses Differentiation and Formation of the Cardiac Chambers. Dev. Dyn. 2004, 229, 763-770. [CrossRef] [PubMed]

25. Ribeiro, I.; Kawakami, Y.; Büscher, D.; Raya, Á.; Rodriguez-León, J.; Morita, M.; Rodríguez Esteban, C.; Izpisúa Belmonte, J.C. Tbx2 and Tbx3 regulate the dynamics of cell proliferation during heart remodeling. PLoS ONE 2007, 2, e398. [CrossRef] [PubMed]

26. Koo, H.Y.; Kume, T. FoxC1-Dependent Regulation of Vascular Endothelial Growth Factor Signaling in Corneal Avascularity. Trends Cardiovasc. Med. 2013, 23, 1-4. [CrossRef] [PubMed]

27. Kidholm, C.L.; Beck, H.C.; Madsen, J.B.; Palstrøm, N.B.; Lindholt, J.S.; Rasmussen, L.M. Preliminary analysis of proteome alterations in non-aneurysmal, internal mammary artery tissue from patients with abdominal aortic aneurysms. PLoS ONE 2018, 13, e0192957. [CrossRef] [PubMed]

28. Hirashiki, A.; Yamada, Y.; Murase, Y.; Suzuki, Y.; Kataoka, H.; Morimoto, Y.; Tajika, T.; Murohara, T.; Yokota, M. Association of Gene Polymorphisms with Coronary Artery Disease in Low- or High-Risk Subjects Defined by Conventional Risk Factors. J. Am. Coll. Cardiol. 2003, 42, 1429-1437. [CrossRef]

29. Pietras, A.; Von Stedingk, K.; Lindgren, D.; Påhlman, S.; Axelson, H. JAG2 induction in hypoxic tumor cells alters notch signaling and enhances endothelial cell tube formation. Mol. Cancer Res. 2011, 9, 626-636. [CrossRef] [PubMed]

30. Pedrosa, A.R.; Trindade, A.; Fernandes, A.C.; Carvalho, C.; Gigante, J.; Tavares, A.T.; Diéguez-Hurtado, R.; Yagita, H.; Adams, R.H.; Duarte, A. Endothelial jagged1 antagonizes Dll4 regulation of endothelial branching and promotes vascular maturation downstream of Dll4/Notch1. Arterioscler. Thromb. Vasc. Biol. 2015, 35, 1134-1146. [CrossRef]

31. Benedito, R.; Roca, C.; Sörensen, I.; Adams, S.; Gossler, A.; Fruttiger, M.; Adams, R.H. The Notch Ligands Dll4 and Jagged1 Have Opposing Effects on Angiogenesis. Cell 2009, 137, 1124-1135. [CrossRef] [PubMed]

32. Vittet, D.; Buchou, T.; Schweitzer, A.; Dejana, E.; Huber, P. Targeted null-mutation in the vascular endothelial-cadherin gene impairs the organization of vascular-like structures in embryoid bodies. Proc. Natl. Acad. Sci. USA 1997, 94, 6273-6278. [CrossRef]

33. Gory-Fauré, S.; Prandini, M.H.; Pointu, H.; Roullot, V.; Pignot-Paintrand, I.; Vernet, M.; Huber, P. Role of vascular endothelialcadherin in vascular morphogenesis. Development 1999, 126, 2093-2102. [CrossRef]

34. Drake, C.J. Embryonic and adult vasculogenesis. Birth Defects Res. Part C Embryo Today Rev. 2003, 69, 73-82. [CrossRef] [PubMed] 
35. Yu, J.; Vodyanik, M.A.; Smuga-Otto, K.; Antosiewicz-Bourget, J.; Frane, J.L.; Tian, S.; Nie, J.; Jonsdottir, G.A.; Ruotti, V.; Stewart, R.; et al. Induced pluripotent stem cell lines derived from human somatic cells. Science 2007, 318, 1917-1920. [CrossRef]

36. Churko, J.M.; Burridge, P.W.; Wu, J.C. Generation of human iPSCs from human peripheral blood mononuclear cells using non-integrative sendai virus in chemically defined conditions. Methods Mol. Biol. 2013, 1036, 81-88. [CrossRef] [PubMed]

37. Berecz, T.; Husvéth-Tóth, M.; Mioulane, M.; Merkely, B.; Apáti, Á.; Földes, G. Generation and Analysis of Pluripotent Stem Cell-Derived Cardiomyocytes and Endothelial Cells for High Content Screening Purposes. In Methods in Molecular Biology; Humana: New York, NY, USA, 2020; Volume 2150.

38. Ontoria-Oviedo, I.; Dorronsoro, A.; Sánchez, R.; Ciria, M.; Gómez-Ferrer, M.; Buigues, M.; Grueso, E.; Tejedor, S.; García-García, F.; González-King, H.; et al. Extracellular Vesicles Secreted by Hypoxic AC10 Cardiomyocytes Modulate Fibroblast Cell Motility. Front. Cardiovasc. Med. 2018, 5, 152. [CrossRef] [PubMed]

39. Robinson, M.D.; McCarthy, D.J.; Smyth, G.K. edgeR: A Bioconductor package for differential expression analysis of digital gene expression data. Bioinformatics 2010, 26, 139-140. [CrossRef] [PubMed]

40. Reimand, J.; Isserlin, R.; Voisin, V.; Kucera, M.; Tannus-Lopes, C.; Rostamianfar, A.; Wadi, L.; Meyer, M.; Wong, J.; Xu, C.; et al. Pathway enrichment analysis and visualization of omics data using g:Profiler, GSEA, Cytoscape and EnrichmentMap. Nat. Protoc. 2019, 14, 482-517. [CrossRef] [PubMed]

41. Raudvere, U.; Kolberg, L.; Kuzmin, I.; Arak, T.; Adler, P.; Peterson, H.; Vilo, J. G:Profiler: A web server for functional enrichment analysis and conversions of gene lists (2019 update). Nucleic Acids Res. 2019, 47, W191-W198. [CrossRef] [PubMed] 